\title{
Diagnostic errors in interactive telepathology
}

\author{
G. Stauch ${ }^{\mathrm{a}, *}$, K.W. Schweppe ${ }^{\mathrm{b}}$ and K. Kayser ${ }^{\mathrm{c}}$ \\ a Department of Pathology, Aurich, Germany \\ ${ }^{\mathrm{b}}$ Department of Gynaecology, Klinikum Ammerland, \\ Westerstede, Germany \\ ${ }^{\mathrm{c}}$ Department of Pathology, Thoraxklinik gGmbH, \\ Heidelberg, Germany
}

Telepathology (TP) as a service in pathology at a distance is now widely used. It is integrated in the daily workflow of numerous pathologists. Meanwhile, in Germany 15 departments of pathology are using the telepathology technique for frozen section service; however, a common recognised quality standard in diagnostic accuracy is still missing. In a first step, the working group Aurich uses a TP system for frozen section service in order to analyse the frequency and sources of errors in TP frozen section diagnoses for evaluating the quality of frozen section slides, the important components of image quality and their influences an diagnostic accuracy. The authors point to the necessity of an optimal training program for all participants in this service in order to reduce the risk of diagnostic errors. In addition, there is need for optimal cooperation of all partners involved in TP service.

Keywords: Telepathology, frozen section service, diagnostic error

\section{Introduction}

Primary frozen section diagnosis and second opinion diagnosis via electronic imaging of histological and cytological slides have become routine work of pathologists and are already integrated in the daily workflow of a pathologist in various departments of diagnostic pathology [2-8].

Some years ago, interactive electronic transfer of images was only used by a small number of exerts because of the need of highly specialised and expensive equipment $[13-15,19]$. The development of new software and hardware components as well as the price reduction enable more pathologists to use this technique especially in primary diagnoses such in frozen section

\footnotetext{
*Corresponding author: Dr. G. Stauch Pathologie Aurich, Wallinghausener Str. 8, 26603 Aurich, Germany. Tel.: +49 4941 65439; Fax: +49 4941 66851; E-mail: info@pathologie-aurich.de.
}

service [15-17]. At present, about 15 departments of pathology in Germany use telepathology in primary histological diagnoses either in the testing phase or in daily work. An increasing rate of publications show individual results; however, these results cannot be compared in between, because generally accepted quality standards are still missing. Therefore, German Society of Pathology has established a working group to evaluate the standards in using electronic imaging technique for frozen section service.

The aim of this investigation is to analyse out results concerning frequency and error sources as first steps to collect data for clinical and technical evaluation of telepathology standards.

\section{Material and method}

In 1994 we established a frozen section service via telepathology (TP) between the Department of Gynaecology, Ammerland Klinikum, Westerstede and the Department of Pathology in Aurich [18]. About 1000 diagnostic procedures, mostly breast lesions, have been performed until now. In this study, 200 consultative cases diagnosed during the period from 04.1998 to 02.1999 were re-evaluated and video images, frozen section slides and slides obtained from paraffin embedded tissue were reviewed, and the errors and the image quality were analysed.

The technical equipment in the Westerstede hospital consists of a microscope (Olympus BH2), a camera (Hitachi HCV 200), a macroscopic camera (JVC TK1070E), a frame grabber (Matrox Millennium) and a PC (Pentium 2). It is connected with the pathology station peer to peer via a commercial network ISDN ( 2 channel $128 \mathrm{~kb} / \mathrm{sec}$ ). The latter is configured with a PC (Pentium 2), and a monitor (EIZO 17' Flex Scan T68), located in the Department of Pathology in Aurich.

Before starting the TP section, the surgeon is asked to send the necessary clinical information: for example, sonography, mammography data and images, etc. to the involved pathologist. The surgeon then selects the suspicious area out of the excised tissue and trans- 
fers a specimen of a volume measuring about $0.7 \times 0.5$ $\times 0.5 \mathrm{~cm}$ to the one-side laboratory. Frozen sections of $5 \mu \mathrm{m}$ thickness stained with Masson Goldner are prepared and positional under the microscope. Overviews of the slides, as well as images of imprint cytology and macroscopy are sent to the pathologist. The pathologist advises by telephone the technician to select a higher magnification and to transfer the selected image. After viewing a number of approximately 8 images the lesion can be diagnosed [10-12].

Images of the TP sessions are stored after setting up a definite diagnosis in a data bank, and the diagnosis is documented. The involved pathologist reviews the frozen section slides and those of paraffin-embedded tissue within 36 hours by conventional microscopy and records the definite diagnosis.

In this study, histological slides obtained from paraffin-embedded tissue are considered to be the gold standard. Diagnostic errors are classified as interpretation errors if differences between TP diagnosis and conventional frozen section diagnosis are obtained, and as sampling errors, if discrepancies between FS slides and histological slides cut from paraffin-embedded tissue are seen. Error qualities were divided into therapeutically irrelevant and therapeutically relevant items depending upon the deviation of dignity of the referred diagnosis.

The quality of each monitor image is scored with 0 4 points concerning motive, brightness and focus. They are summarised and graded in 1-5 for insufficient, in 6-7 for good and in 8-9 for excellent quality. From 200 consecutive cases, 126 benign and 69 malignant breast lesions are analysed excluding 5 frozen sections from the ovary and peritoneum in two additional patients (Table 1).

Table 1

200 Frozen section examinations via telepathology 4/98-2/99

\begin{tabular}{llcc}
\hline Organ & Dignity & $\begin{array}{c}\text { Examination } \\
n\end{array}$ & $\begin{array}{c}\text { Pt. } \\
n\end{array}$ \\
\hline Mamma & benign & 126 & 103 \\
& malignant & 69 & 63 \\
Peritoneum/ & benign & 4 & 1 \\
Ovary & malignant & 1 & 1 \\
Total & & 200 & 168 \\
\hline
\end{tabular}

The discrepancy between number of examinations and the number of patients can be explained by multiple examinations of the same lesion.

\section{Results}

Relevant diagnostic errors comprising 6 false negative and 9 referred diagnoses were seen in 15 cases of this series. The authors did not find any false positive diagnosis. Referred diagnoses were mostly observed in cases with invasive lobular carcinoma or invasive ductal carcinoma with predominant intraductal compartments. Two cases with diffuse lobular carcinoma sampling errors were observed, which were caused by surgeons who cut the lesions and who were not familiar with the rather benign macroscopic aspect of the specimens (Table 2).

An example of an imprint image and a histological images is given in Figs 1 and 2.

A total of 1234 monitor images was reviewed and scored according to the image qualities (Fig. 3): $40 \%$ of macroscopic images, and $20 \%$ of histological respectively $15 \%$ of cytological images were scored $0-5$, i.e., found to be of insufficient quality.

The analysis of 400 insufficient macroscopic and microscopic monitor images (Fig. 4) demonstrates: (a) a loss of information due to the selection of wrong

Table 2

Frequency of diagnostic errors in 195 breast lesions

\begin{tabular}{lcccc}
\hline Dignity & Cases & Non-relevant & False negative & Referred \\
& $n$ & $n$ & $n$ & $n$ \\
\hline Benign & 126 & 32 & - & 4 \\
Malignant & 69 & 8 & 6 & 5 \\
Total & 195 & $40(20.5 \%)$ & $6(3.6 \%)$ & $9(4.6 \%)$
\end{tabular}

Therapeutic relevant errors (false negative + referred diagnoses) $=$ $8.2 \%$ of all cases.

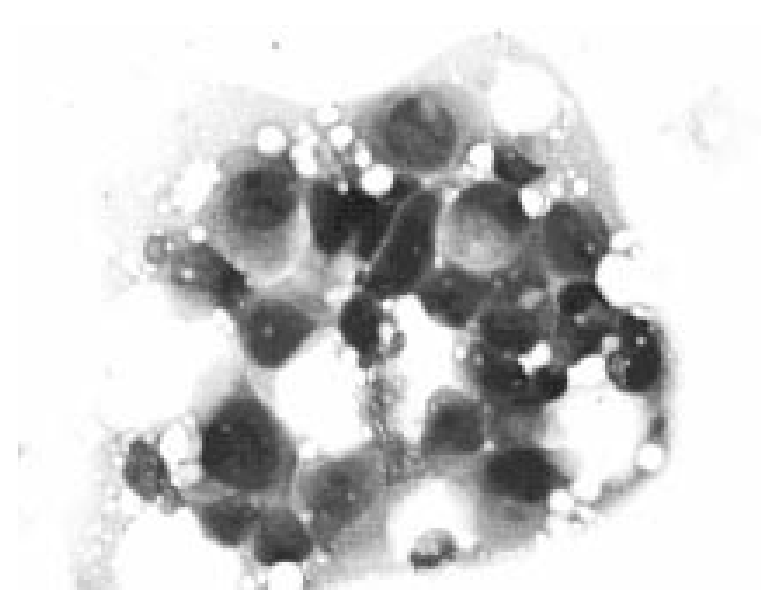

Fig. 1. Cluster of ductal carcinoma cells MG stain; $20 \times 3$-chip camera JPG compression $1: 60$. 
Table 3

Diagnostic accuracy in TP. Frozen section service

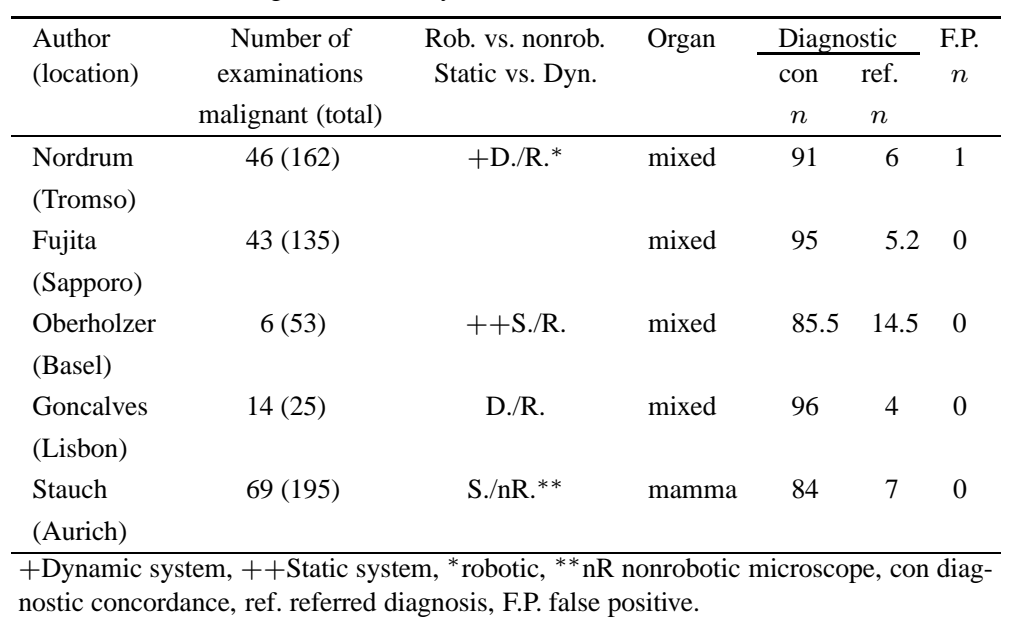
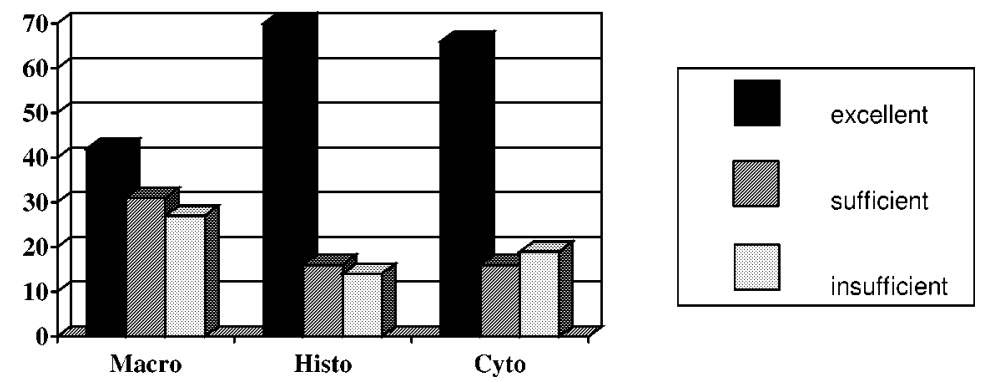

Fig. 3. Distribution of 1234 TP images in quality categories regarding macroscopy, histology and cytology (viewed images in percent).

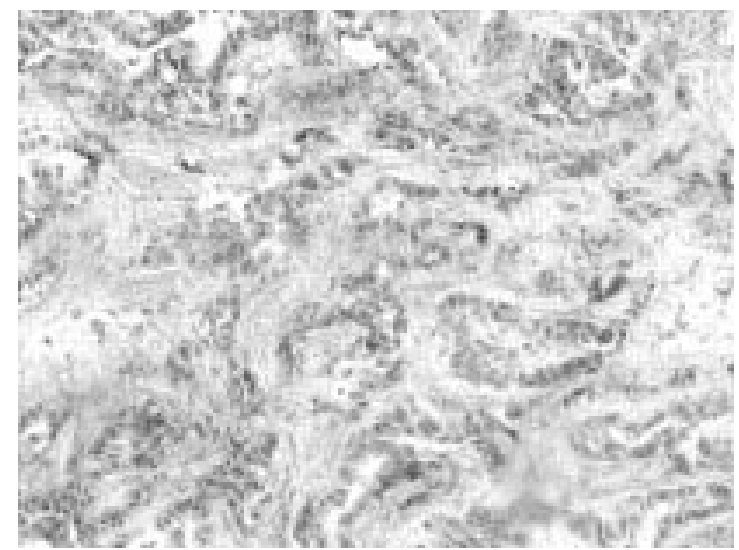

Fig. 2. Ductal carcinoma MG stain $\times 10$; 3-chip camera JPG compression $1: 20$.

areas in the slide (sampling error), (b) errors in focusing the camera of macroscopic specimens or mistakes in handling the microscope, and (c) histological image errors caused by inadequate illumination due to mismanagement of the camera mounted to the micro- scope (Fig. 5).

Reviewing the quality of frozen section slides, and estimating the thickness, coherence of specimen and quality of stains, more than $40 \%$ of TP errors are caused by a low image quality of the frozen section slides (Figs 5 and 6).

\section{Discussion}

The results of the working group Aurich are comparable with those published by Oberholzer et al. 1996 [16]. Other groups found a higher diagnostic concordance, for example, published Goncalves et al. by 1995 [4] or Kayser et al. [11,12].

These differences can be explained by different TP procedures. Most of the studies use remote controlled microscopes which enable the pathologist to view the complete slide and to select the suspicious areas himself. In contrast, our system is based on non-robotic microscope and a technician selects the suspicious areas guided by acoustic commands of the pathologist. 


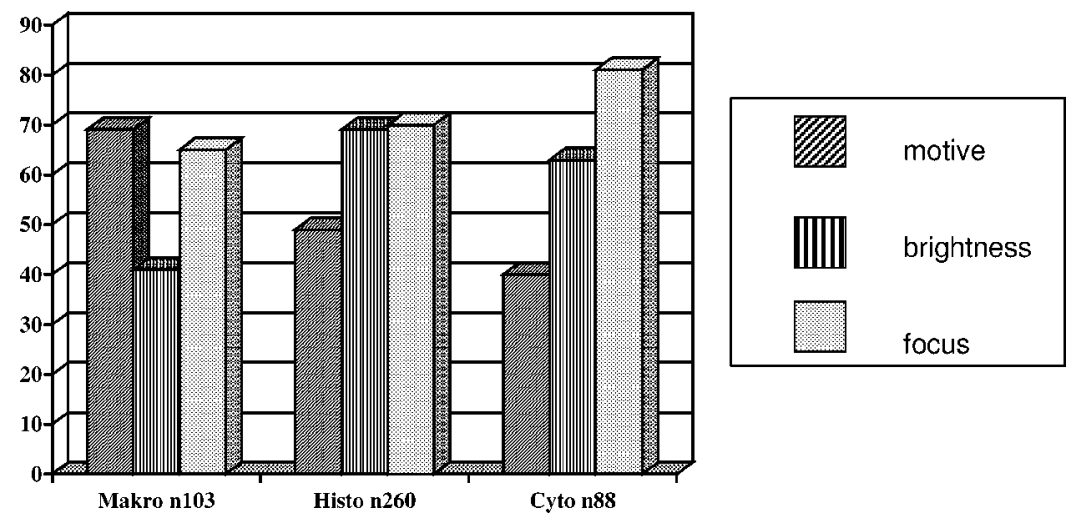

Fig. 4. Error analyses of 400 non-optimal images in percent of viewed images evaluating insufficient quality criteria motive, brightness and focus.
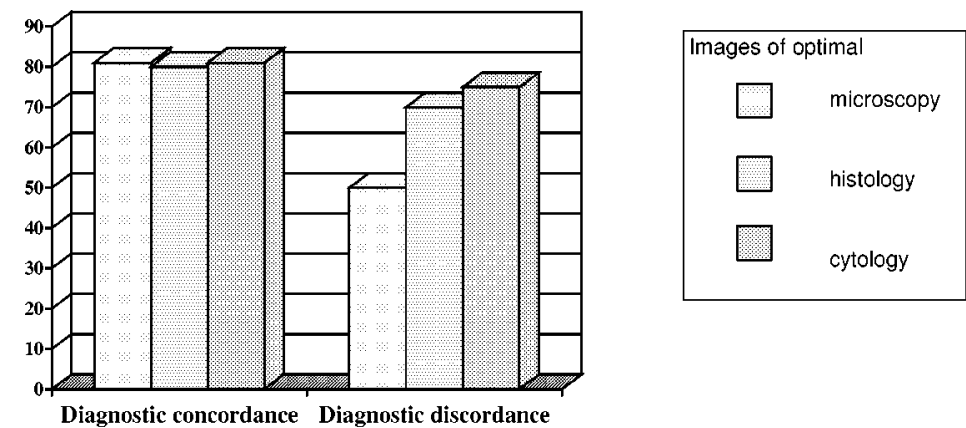

Fig. 5. Relation of excellent image quality of macroscopy, histology and cytology in correct diagnoses versus false or referred diagnoses (in percent of analyzed cases).
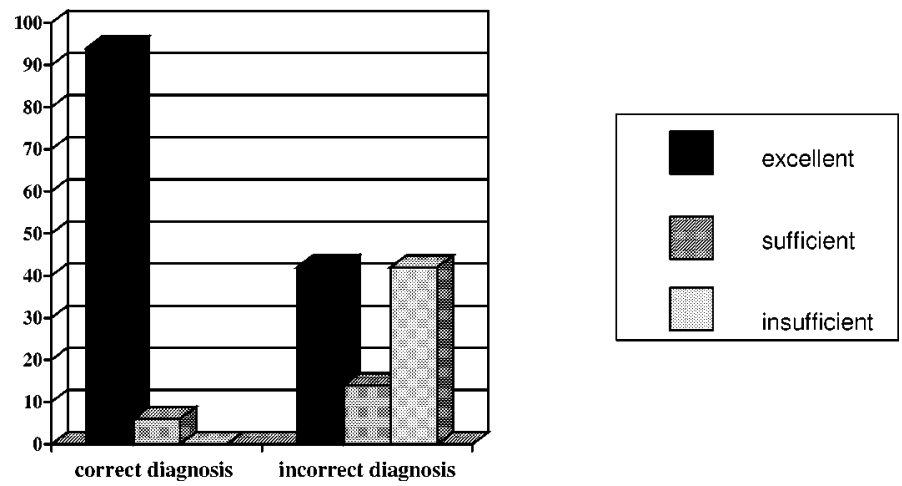

Fig. 6. Relation between frozen section slide quality in diagnostic correct cases versus incorrectly diagnosed cases (in percent of analyzed cases).

In this procedure the viewing fields are narrowed and visual sampling errors occur more frequently.

When using a remote control microscope the diagnostic time is longer in comparison to our system [16]. The time needed for diagnosis was not published by other teams, however, this time should be taken into consideration when evaluating the quality of frozen section services, and should be added to the rate of se- lection errors.

The diagnostic accuracy is strongly related to the quality of monitor images. A 24 bit color depth even in relatively monochrome Masson Goldner stain is mandatory. Occasionally transmitted images in 16 bit colour depth were of minor quality in both histology and macroscopy. A color reduction to 16 bits may be used in order to minimise the data volumes without 
loss of information. Our results differ from those published by Dolittle who recommended a 6 bits color depth as sufficient for TP procedures [1].

The image quality of frozen section slides in telepathology performance is more important compared to that of conventional frozen section service [9]. Reviewing the slides we found that $60 \%$ of the wrong diagnosed cases revealed a poor quality image of the frozen section slides in contrast to $95 \%$ of the correctly diagnosed cases which displayed either a sufficient or excellent image quality.

The insufficient image quality of the frozen sections is mainly caused by increased thickness, torn areas or weekly respectively insufficiently stained preparations. In conventional frozen section service the pathologist reads the slides in a dynamic process permanently going through and controlling brightness, creating a puzzle image out of separated fragments. In contrast, electronic images, cannot be manipulated in this manner and quality errors can be influence remarkable the monitor images. Thus, the reading pathologist gets the impression that electronic imaging gains the defects of the cutting procedures.

The second factor in creating diagnostic deviation is related to the lack of information when evaluating the macroscopic image. One of the diagnostic principles in frozen section service is that the macroscopic inspection and palpation of a specimen should not be separated from the microscopic evaluation of the frozen section slides. Concordance of macroscopic and microscopic procedures guarantees the best standard of diagnostic quality.

In our series, only $30 \%$ of macroscopic images were judged to be of good or excellent quality. The other cases presented either with inadequate motive collection or with non-optimum image focus. The loss of image quality was caused by different surface structures of the specimen, the use of an one-chip camera for macroscopy and the system's immanent loss of image details due to presentation on a video monitor with a limited pixel resolution. Focusing a three-dimensional object in a single image can cause blurring artefacts. In addition, the pathologist has no opportunity to palpate the specimen, and sampling information or indications for the dignity of lesions are missing. Therefore, our results show the need for additional information in frozen section diagnosis.

Clinical data, image of mammography and sonography can be used to compensate the lack of macroscopic and palpative information.

\section{Conclusion}

Approximately additional 10\% referred respectively false negative diagnoses in breast lesions compared to the results of conventional frozen section diagnoses may be considered to be tolerable regarding the advantages of this technique: Offering frozen section service to surgical departments as a fast intra-operative procedure with the advantages of saving transportation costs and of reducing time of anaesthesia.

The rate of referred diagnosis in our system is still high, and there is an ethic demand to reduce this rate.

In our study, the most important factors which are associated with the diagnostic accuracy of telepathology frozen section service rely on the insufficient quality of frozen sections with low image quality, and handling problems in the use of the microscope and the camera by the technician.

The lack of information from the inspection and palpation of the surgical specimen is of additional importance.

Technical aspects such as poor image quality or use of a non-robotic microscope seem to be of minor influence on the diagnostic accuracy.

These results demonstrate a high demand for both an optimal training of the technical staff at the station positioned in the hospital (department of surgery) and an optimum information transfer prior to the telepathology sessions between the surgeon and the pathologist.

\section{References}

[1] M.H. Doolittle, K.W. Doolittle, Z. Winkelmann and D.S. Weinberg, Color images in telepathology: how many colours do we need?, Hum. Pathol. 28 (1997), 36-41.

[2] T.J. Eide and I. Nordrum, Current status of telepathology (Review article), APMIS 102 (1994), 881-890.

[3] M. Fujita, Y. Suzuki, M. Takahashi, K. Tsukamoto and K. Nagashima, The validity of intraoperative frozen section diagnosis bases an video-microscopy (telepathology), Gen. Diagn. Pathol. 141 (1995), 105-110.

[4] L. Goncalves and C. Cunha, One year experience with telepathology for frozen sections, Elec. J. Pathol. Histol. (1995), No. 954-09.

[5] B.E. Halliday, A.K. Bhattacharyya, A.R. Graham, J.R. Davis, S.A. Leavitt, R.B. Nagle, W.J. McLaughlin, R.A. Rivas, R. Martinez, E.A. Krupinski and R.S. Weinstein, Diagnostic accuracy of an international statio-imaging telepathology consultation service, Hum. Pathol. 28 (1997), 17-21.

[6] K. Kayser, M. Drlicek and W. Rahn, Aids of telepathology in intraoperative histomorphological tumor diagnosis and classification, In vivo 7 (1993), 379-382. 
[7] K. Kayser and M. Drlicek, Visual telecommunication for expert consultation of intraoperative sections, Zentralbl. Pathol. 138 (1992), 381-436.

[8] K. Kayser, P. Fritz and M. Drlicek, Aspects of telepathology in routinary diagnostic work with specific emphasis an ISDN, Arch. Anat. Cytol. Pathol. 43 (1995), 216-218.

[9] K. Kayser and G. Kayser, Telepathology - aspects of social influence and quality control, Elec. J. Pathol. Histol. (1996), No. 963-04.

[10] K. Kayser, G. Kayser and S. Zink, New technical aspects in telepathology, Elec. J. Pathol. Histol. (2000), No. 003-04.

[11] K. Kayser, M. Oberholzer, G. Weisse, I. Weisse and H. v. Eberstein, Long distance image transfer: First results for its use in histopathological diagnosis, Acta Pathol. Microbiol. Immunol. Scand. 99 (1991), 808-814.

[12] K. Kayser, J. Szymas and R. Weinstein, Telepathology Telecommunication, Electronic Education and Publication in Pathology, Springer, Heidelberg, New York, 1999.

[13] K. Kayser and G. Kayser, Basic aspects of and recent development of telepathology in Europe with specific emphasis an quality assurance, J. Anal. Quant. Cytol. Histol. 21 (1999), 319-328.
[14] K. Kayser, Telemedizin, Wiener Klin. Wschr. 108 (1996), 932940.

[15] K. Kayser, Telepathology in Europe. Its practical use, Arch. Anat. Cytol. Pathol. 43 (1995), 196-199.

[16] M. Oberholzer, H.R. Fischer, H. Christen, S. Gerber, M. Brühlmann, M. Mihatsch, T. Gahm, M. Famos, C. Winkler, P. Fehr, H.J. Hosch and L. Bächtold, Telepathology: frozen section diagnosis at a distance, Virchows Arch. 426 (1995), 3-9.

[17] P. Schwarzmann, B. Binder, M. Käser and R. Klose, European Field Tests with Histkom Telepathology Equipment, The Impact of Telemedicine an Health Care Management, M. Nerlich and R. Kretschmer, eds, IOS Press, 1999.

[18] G. Stauch and K.W. Schweppe, One year experience with telepathology for frozen selections, Elec. J. Pathol. Histol. (1995), No. 954-08.

[19] R.S. Weinstein, A.K. Bhattacharyya, A.R. Graham and J.R. Davis, Telepathology: a ten-year progress report, Hum. Pathol. 28 (1997), 1-7. 


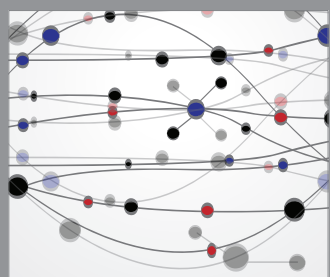

The Scientific World Journal
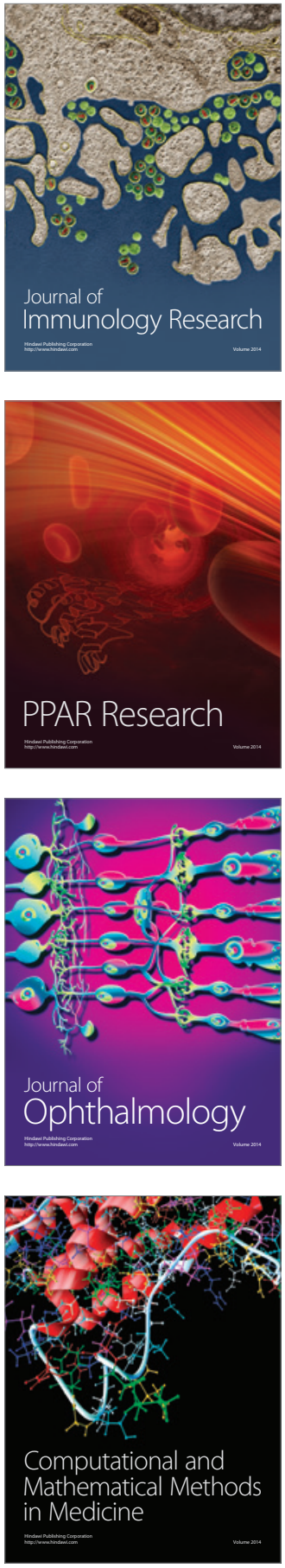

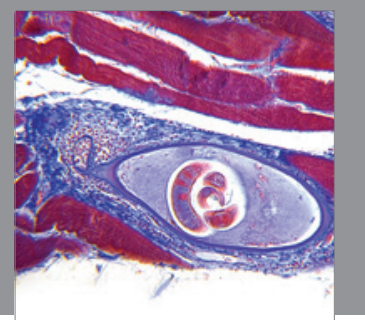

Gastroenterology

Research and Practice
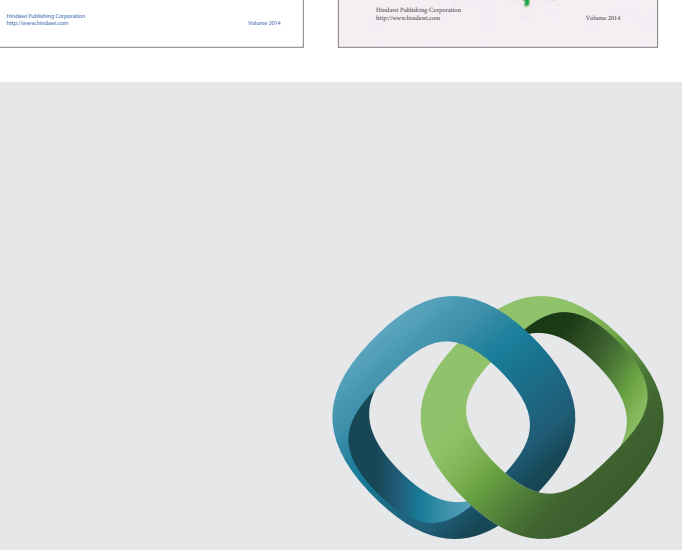

\section{Hindawi}

Submit your manuscripts at

http://www.hindawi.com
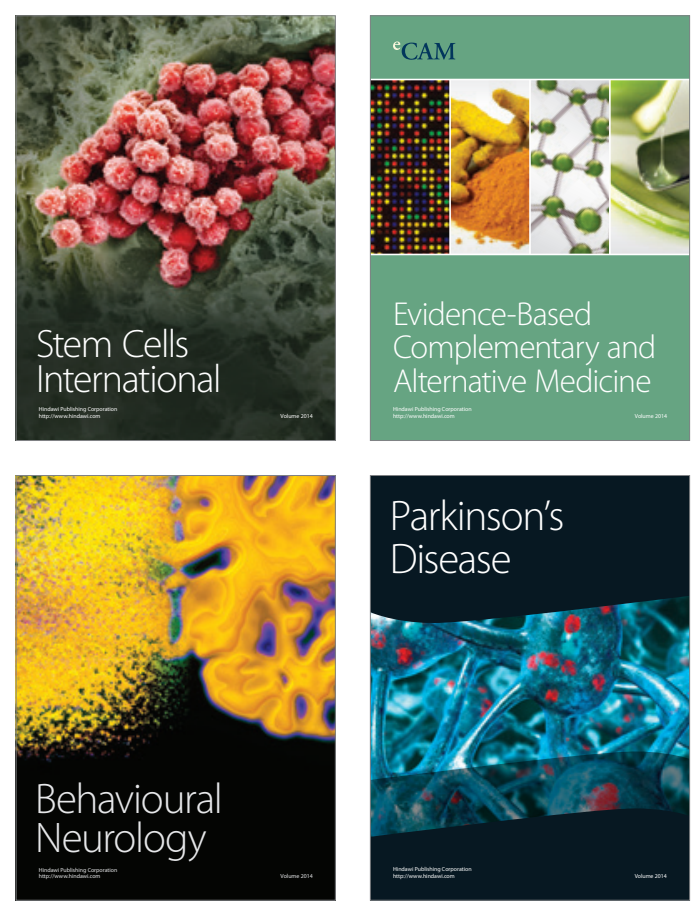

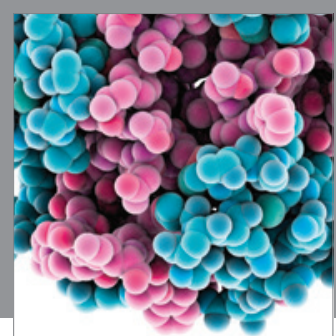

Journal of
Diabetes Research

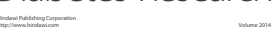

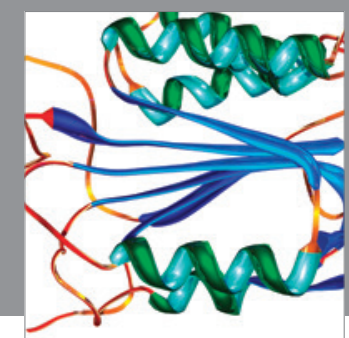

Disease Markers
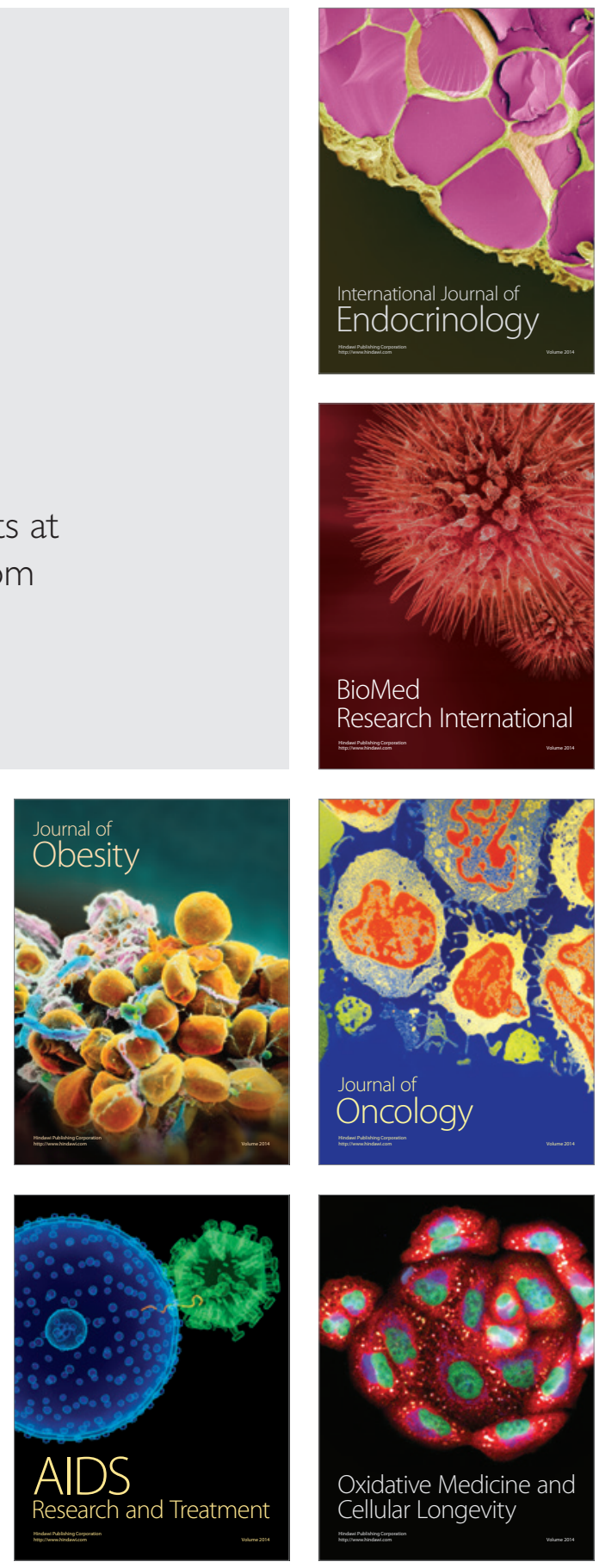